\section{Gelungenes Werk zur Molekularen Allergiediagnostik}

D ie Molekulare Allergiediagnostik hat die Laboranalytik für Typ-1-Allergien in den vergangenen Jahren auf eine völlig neue Qualitätsstufe gehoben. Wurden früher nahezu ausschließlich Allergenextrakte zur Diagnostik verwendet, die zahlreiche allergene und nichtallergene Komponenten enthalten, ist die In-vitro-Diagnostik durch Verwendung molekular definierter Allergenkomponenten sehr viel zielgerichteter. Tatsächlich können hierdurch die eigentlich krankheitsauslösenden Proteine detektiert werden. Auch und insbesondere für HNO-Ärzte kann die Diagnostik hierdurch wesentlich zielgerichteter und exakter durchgeführt werden.

Somit ist die Auseinandersetzung mit den in diesem Buch aufgeworfenen Fra- gestellungen für die HNO-Ärzte immens wichtig. Die bekannten Experten Jörg Kleine-Tebbe und Thilo Jakob bürgen als Mitautoren und Herausgeber für ein qualitativ hochstehendes Buchprojekt.

Alle Leser dieses umfassenden Werkes werden von Konzept und Ausführung begeistert sein. Die klare didaktische Struktur verschafft einen raschen Überblick über die theoretischen Grundlagen und die wichtigsten klinisch-praktischen Gebiete der Molekularen Allergiediagnostik. Dabei erhält der Leser zahlreiche praktische Tipps aus der langjährigen Erfahrung der Autoren und Herausgeber und umfassende Literaturhinweise. Zahlreiche gute Tabellen und Zeichnungen begleiten den Text. Beson-
J. Kleine-Tebbe, T. Jakob

Molekulare Aller-

Molekulare

Allergiegiediagnostik

diagnostik

392 Seiten

Springer-Verlag 2015

ISBN 978-3-662-45221-9

Hardcover $€ 49,99$

ders gelungen und geeignet für HNOÄrzte erscheinen die Kapitel zu „Proteinfamilien“, „Das Konzept der PollenPanallergene“ und „Molekulare Allergiediagnostik mit Singleplex- und Multiplex-Verfahren“.

Insgesamt ein außergewöhnlich gelungenes Werk, das sicherlich zahlreiche interessierte Leser im Kreis der allergologisch tätigen Ärzte finden wird und daher insbesondere auch für die HNOÄrzte eine wertvolle Bereicherung sein wird.

Prof. L. Klimek

\title{
Gleichgewichtsstörungen im Überblick
}

D as Lehrbuch gibt zunächst einen umfassenden Überblick über Anatomie und Physiologie des Gleichgewichtssystems. Dadurch unterscheidet es sich nicht von anderen Büchern namhafter deutscher Neurootologen, ist jedoch sehr verständlich, auch für Einsteiger geschrieben und mit guten Zeichnungen versehen. Der Leser erkennt die komplexe Verschaltung innerhalb des vestibulären Sinns und anderer Sinne wie Sehen, Hören, Fühlen. Bei Überforderung des Systems oder falsch interpretierten Meldungen aus anderen Sinnesbereichen können physiologische Störungen auftreten, z.B. Reisekrankheit, oder organische Störungen, die wegen der vielen Verschaltungen ein sehr buntes Bild an Symptomen aufweisen, die vom Patienten als Schwindel bezeichnet werden.

Ernst und Basta haben hervorgehoben, dass ein Patient, der über Schwindel klagt, ganzheitlich betrachtet werden muss, und haben deshalb folgerichtig Koautoren einbezogen, vorwiegend Spezialisten auf dem Gebiet der Neurologie, Neuroradiologie, Kardiologie und Psy- chosomatik. Die Autoren haben Störungen entsprechend ihrer Lokalisation benannt und besonderen Wert auf eine gute Darstellung von Untersuchungsmethoden gelegt. Hierzu gehören Untersuchungen am Krankenbett, aufwendige Untersuchungen des Otolithen- und Bogengangsystems und auch eine Bewertung zervikaler Störungen nach Traumen sowie neurologische, psychische, kardiologische und ophthalmologische Störungen. Manche Ursachen wurden erst in letzter Zeit erforscht und in der zweiten erweiterten Auflage der "Gleichgewichtsstörungen“ umfassend ergänzt. Parallel dazu hat sich auch das therapeutische Spektrum deutlich erweitert. Es werden umfassend alle peripher-vestibulären und zentral-verstibulären Störungen von Kindes- bis Seniorenalter beschrieben, auch bei internistischen, ophthalmologischen, zervikalen oder somatoformen Erkrankungen, und jeweils entsprechende Behandlungsmöglichkeiten empfohlen. Besonderer Wert wird auf Rehabilitationstechniken nach vestibulären Störungen sowie auf die Sturzprophylaxe für eine alternde Bevölkerung gelegt, und es werden

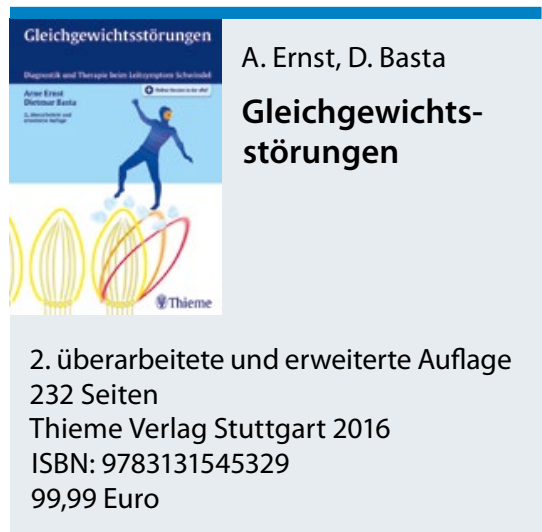

auch seltenere Krankheitsbilder, die nach Traumen entstehen können, z.B. das Bogengangsdehiszenzsyndrom, erläutert.

Jeder Arzt, der Patienten behandelt, die über Schwindel oder den „Zustand des Anderssein“ klagen, sollte dieses Buch besitzen. Aufgrund der verständlichen Ausführungen mit sehr guten Abbildungen, untermauert durch sehr übersichtliche Merksätze, ist es auch für Einsteiger geeignet, des Weiteren für erfahrene Ärzte, die Gleichgewichtsstörungen begutachten, da diesem Thema ein Extrakapitel gewidmet ist.

Prof. Karin Schorn 